\title{
Coronavirus Disease (COVID-19) and the Gastrointestinal System in Children
}

\author{
John Matthai ${ }^{1}$, Naresh Shanmugam ${ }^{2}$ and Prasanth Sobhan ${ }^{3}$ for the Indian Society OF Pediatric \\ Gastroenterology, Hepatology and Nutrition; Pediatric Gastroenterology Chapter of Indian Academy \\ of Pediatrics \\ From ${ }^{1}$ Masonic Medical Centre, Coimbatore, Tamil Nadu; ${ }^{2}$ DrRela Institute and Medical Center, Chennai, Tamil Nadu; and, \\ ${ }^{3}$ Government Medical College, Thiruvananthapuram, Kerala; India. \\ Correspondence to: Prof John Matthai, Pediatric Gastroenterologist, Masonic Medical center, Race course, Coimbatore 641004, \\ Tamil Nadu,India.psg_peds@yahoo.com
}

\begin{abstract}
Severe acute respiratory syndrome coronavirus 2 (SARS-CoV2), though primarily a respiratory pathogen, also involves the gastrointestinal tract. Similar to the respiratory mucosa, angiotensin converting enzyme-2 (ACE-2) receptor and transmembrane serine protease 2 (TMPRSS2) co-express in the gastrointestinal tract, which facilitates viral entry into the tissue. Less than $10 \%$ of children with infection develop diarrhea and vomiting. Prolonged RT PCR positivity in the stool has raised the possibility of feco-oral transmission. Elevated transaminases are common, especially in those with severe coronavirus disease (COVID-19). Children with inflammatory bowel disease and post liver transplant patients do not have an increased risk of disease, and should remain on medications they are already on. Children with chronic liver disease should continue their medications as usual. All elective procedures like endoscopy should be postponed.
\end{abstract}

Keywords: Diarrhea, Fecal shedding, Inflammatory bowel disease, Liver transplant, SARSCoV-2.

I $\mathrm{n}$ Severe acute respiratory syndrome corona virus 2 (SARS-CoV2) infection the respiratory system is the main target organ; however, the gastrointestinal tract and the liver may also be involved, either symptomatically or with only laboratory derangements. The virus has been detected in respiratory secretions, feces and blood.

\section{COVID-19 AND THE INTESTINE}

\section{Fecal-Oral Transmission}

The virus attaches to the angiotensin converting enzyme2 (ACE 2) receptors of the intestine. Among 73 adult patients, 39 tested positive for RNA in stool samples and 17 remained positive for the virus in stool even after becoming negative in respiratory samples [1]. $\mathrm{Xu}$, et al. [2] reported that 8 of their 10 pediatric patients were positive for RT-PCR in rectal swabs, which remained detectable well after nasopharyngeal swabs turned negative. Thus fecal oral transmission is being proposed as another route of spread of infection and it is possible that even asymptomatic patients may be shedding the virus in the stool. However, till date there is only report of the virus cultured from a single stool specimen. At present it is unclear whether the persistence of RNA in the stool is secondary to its continued positivity in bronchoalveolar lavage, even when nasopharyngeal mucosa swabs are negative. While more data, particularly on fecal infectivity are awaited, this finding has grave implications for developing countries [1,2].

\section{Gastrointestinal Manifestations}

Some children have reported nausea, vomiting, diarrhea and abdominal pain during the course of the disease. Diarrhea most often occurred 1 to 8 days after the onset, with a median time of 3.3 days. Some patients had watery diarrhea as the first symptom. Lu,et al. [3] have reported that diarrhea and vomiting were observed in $15(8.8 \%)$ and $11(6.4 \%)$ in a cohort of 171 children. While Jin, et al. [4] reported that the presence of gastrointestinal symptoms in adults was associated with more severe illness, there is no similar report in children.

Intestinal involvement has many reasons. Angiotensin converting enzyme 2 (ACE2) receptor and transmembrane serine protease 2 (TMPRSS2) are key proteins in the cell entry process of the virus. Coexpression of these two proteins in the same cell is critical for viral entry. Like alveolar type II cells in the lung, gland cells of the esophagus and absorptive enterocytes in ileum and colon express them together. After viral entry, virus-specific RNA and proteins are synthesized in the cytoplasm of these cells to assemble new virions, which are released to gastrointestinal tract. This 
gastrointestinal tropism explains the digestive symptoms and the viral shedding in stool. Xiao, et al. [5] have given more convincing evidence of primary gastrointestinal tract involvement in one patient using endoscopic biopsies. They observed that in more than $20 \%$ of their adult patients, the viral RNA remained positive in the stool even after it became negative in the respiratory tract. They have recommended that RT PCR of the stool should be performed before deciding that a hospitalized patient is not infective. The virus also seems to alter the intestinal flora, even when only the respiratory mucosa is involved, through the common mucosal immune system regulation called the 'gut-lung axis' [6].

Available evidence suggests that IBD (inflammatory bowel disease) patients do not have an increased risk of developing COVID-19 and should stay on IBD medications. Surrogate markers of inflammation (ESR, CRP, patient-reported outcomes) may be an alternative to face-to-face office visits during the epidemic, especially for those in remission. Newly diagnosed patients should be treated according to the standard protocols as before the epidemic. There is currently no evidence that any of the drugs used in IBD including immune-modulators and biologicals increases the severity of COVID and the risk of a disease flare outweighs any estimated risk of SARSCoV2 infection. Corticosteroids can be used to treat disease relapses, but as always recommended in children, the drug should be weaned as soon as possible. The use of anti-tumor necrosis factor drugs should be continued as earlier, while making sure that infusion centers in hospitals take standard prevention measures of COVID. Switching from infliximab to adalimumab in a stable child should be discouraged unless impossible to provide intravenous infusions, considering the higher risk of disease exacerbation.

All routine endoscopic procedures must be avoided, since they are aerosol generating, more so in children where it also involves airway management. The upper gastrointestinal endoscopy carries a higher risk of aerosols than lower gastrointestinal endoscopy. Acute upper or lower gastrointestinal bleeding, esophageal obstruction, foreign body ingestion etc. may require endoscopy without delay, but should be done with full personal protection equipment including the N95 mask. Biopsies must be placed in formalin immediately. Endoscopy room and disinfection policy should be followed as per standard protocol $[7,8]$.

\section{COVID AND THE LIVER}

A mild rise in transaminases is common with COVID-19 disease but serious liver dysfunction is uncommon. The elevated transaminases are often accompanied by high creatine kinase and lactate dehydrogenase suggesting the possibility that viral myositis may also be the cause. Elevated liver enzymes are more common in those with severe spectrum of the disease (40-60\%), compared to those who are asymptomatic or have mild disease (18$25 \%$ ). Bilirubin levels are also more than double in those with severe infection, when compared to those with milder disease. This is probably related to virus triggered auto reactive $\mathrm{T}$ cells and cytokine storm. Though hepatocytes and biliary epithelium are abundant in angiotensin-converting enzyme 2 (ACE2) receptors, which is the same receptor that the virus uses for entering the lungs, there is no evidence of active replication of the virus in hepatocytes. Hypoxic injury from respiratory distress and drug induced liver injury (remdesivir, tocilizumab) are other possible causes for the abnormal liver function tests in patients [9].

Any child with COVID-19 disease and raised transaminases should be investigated for other causes of liver disease. For patients who are asymptomatic or have only mild disease, hospital visit is unnecessary and a teleor video-consultation is sufficient. Newly diagnosed patients with jaundice, aspartate amino transferase/ alanine amino transferase $>500 \mathrm{IU} / \mathrm{L}$ or recent onset hepatic decompensation should be evaluated in hospital. At present, there is no concrete evidence to show that COVID-19 co-infection causes significant worsening in of underlying chronic liver disease. However, evidence from the previous SARS $\mathrm{CoV}$ epidemic suggests otherwise, but more data is required. Elevated transaminases in COVID-19 disease are not a contraindication for antiviral therapy, with regular monitoring of liver function [10].

Post-liver transplant patients need particular emphasis on preventive measures like frequent hand washing, cleaning frequently touched surfaces and social distancing etc. As the cell injury in COVID-19 disease is thought to be immune-mediated, immunosuppression and mycophenolate should not be reduced or stopped in asymptomatic post-transplant patients. In an established COVID-19 infection, continue calcineurin inhibitors targeting a lower trough levels and lower the dose of mycophenolate or azathioprine. Patients on high dose steroids should have it reduced to a minimum dose based on body weight to prevent adrenal insufficiency. At present there is no recommendation for any antivirals or hydroxychloroquine prophylaxis either in post-liver transplant children or those with COVID-19 associated acute liver disease.

Children on treatment for chronic liver diseases like Wilson disease, autoimmune hepatitis, hepatitis B and C 


\section{Key Messages}

- There is gastrointestinal mucosal involvement in COVID-19 and viral RNA is detected in the stool even after the nasopharyngeal swabs are negative.

- Elevated transaminases are common in severe disease, but serious liver dysfunction is uncommon.

- Routine endoscopies should be avoided during the epidemic, since it is an aerosol-generating procedure.

- Children with inflammatory bowel disease, chronic liver disease and post liver transplant patients do not seem to have an increased risk of disease and should continue their medications.

should continue their treatment protocols. All elective liver transplantations should be postponed.

Contributors: JM: as chairman coordinated and edited the paper; NS authored the segment on Liver; PS the segment on intestine. All authors participated in finalizing the paper.

Funding: None; Competing interests: None stated.

\section{REFERENCES}

1. Hindson J. COVID-19: Faecal-oral transmission? Nature Rev Gastroenterol Hepatol. 2020. Available from https:// www.nature.com/nrgastro/s41575-020-0295-7. Accessed March 30, 2020.

2. $\mathrm{Xu} \mathrm{Y,} \mathrm{Li} \mathrm{X,} \mathrm{Zhu} \mathrm{B,} \mathrm{Liang} \mathrm{H}$, Fang $\mathrm{C}$, Gong $\mathrm{Y}$,et al. Characteristics of pediatric SARS-CoV-2 infection and potential evidence for persistent fecal viral shedding. Nature Med.2020. Available from:https://www.nature. com/naturemedicine/s41591-020-08174. Accessed March 15,2020 .

3. Lu X, Zhang L, Du H, Zhang J, Li YY, Qu J,et al. SARSCoV-2 infection in children. New England J Med. 2020. Available from: https://www.nejm.org/NEJMc2005073. Accessed March 29, 2020.

4. Jin X, Lian J-S , Hu JH, Gao J, Zheng L, Zhang YM,et al. Epidemiological, clinical and virological characteristics of 74 cases of corona virus-infected disease 2019 (COVID19) with gastrointestinal symptoms. Gut.2020. Available from:https://gut.bmj.com/gutjnl-2020- 320926. Accessed March 20, 2020.

5. Xiao F, Tang M, Zheng X, Liu Y, Li X, Shan H.Evidence for gastrointestinal infection of SARS-CoV-2.
Gastroenterology. 2020. Available from: https://www. gastrojournal.org/j.gastro.2020.02.05. Accessed March 27, 2020.

6. Pan L, Mu M, Ren HG, Yang P, Sun Y, Wang R,et al.Clinical characteristics of COVID-19 patients with digestive symptoms in Hubei, China: A descriptive, crosssectional, multicenter study. Am J Gastroenterol. 2020. Available from: https://journals.lww.com/ajg/ajg. 0000000000000620 . Accessed March 31, 2020.

7. Danese S, Cecconi M, Spinelli A. Management of IBD during the COVID-19 outbreak: Resetting clinical priorities. Nature Rev Gastroenterol Hepatol.2020. Available from:https://www.nature.com/nrgastro/s41575020-0294-8. Accessed March 30, 2020.

8. Mao R, Liang J, Shen J, Ghosh S, Zhu LR, Yang H,et al on behalf of the Chinese Society of IBD, Chinese Elite IBD Union, and Chinese IBD Quality Care Evaluation Center Committee. Implications of COVID-19 for patients with pre-existing digestive diseases. Lancet Gastroenterol Hepatol. 2020. Available from: https://www.the lancet.com llangas/S2468-1253(20)30076-5. Accessed March 30, 2020.

9. Zhang c, Shi 1, Wang F-S. Liver injury in COVID-19: Management and challenges. Lancet Gastroenterol Hepatol. 2020. Available from: https://www.thelancet. com/gastrohepS246812532030057-1. Accessed April 06, 2020.

10. Bangash MN, Patel J, Patrekh D. COVID-19 and the liver: Little cause for concern. Lancet GastroenterolHepatol. 2020. https://www.thelancet.com/gastrohepS2468-1253 (20)30084-4. Accessed April 06, 2020. 